\title{
LncRNA XIST serves as a diagnostic biomarker in gestational diabetes mellitus and its regulatory effect on trophoblast cell via miR-497-5p/FOXO1 axis
}

\author{
Yanchuan $\mathrm{Li}^{1}$, Xiaohua Yuan ${ }^{2}$, Ziyun Shi ${ }^{1}$, Haili Wang ${ }^{1}$, Duomei Ren ${ }^{1}$, Ya Zhang ${ }^{1}$, Yangyang Fan ${ }^{1}$, \\ Yanfeng Liu ${ }^{3}$, Zhangxia Cui ${ }^{4}$
}

${ }^{1}$ Department of Obstetrics, Shaanxi Provincial People's Hospital, Xi'an, China; ${ }^{2}$ Department of Obstetrics and Gynecology, Shaanxi Provincial People's Hospital, Xi'an, China; ${ }^{3}$ Department of General Surgery, The Second Affiliated Hospital, Xi'an Medical University, Xi'an, China; ${ }^{4}$ Department of Obstetrics, The Second Affiliated Hospital of Shaanxi University of Chinese Medicine (Xi Xian Central Hospital), Xianyang, China Contributions: (I) Conception and design: Y Li; (II) Administrative support: Z Cui; (III) Provision of study materials or patients: X Yuan, Z Shi; (IV) Collection and assembly of data: H Wang; (V) Data analysis and interpretation: D Ren, Y Zhang, Y Fan, Y Liu; (VI) Manuscript writing: All authors; (VII) Final approval of manuscript: All authors.

Correspondence to: Zhangxia Cui. Department of Obstetrics, The Second Affiliated Hospital of Shaanxi University of Chinese Medicine (Xi Xian Central Hospital), No. 831 Longtaiguan Road, Fengxi New Town, Xixian New District, Xianyang 712000, China. Email: czmi103259@163.com.

Background: Gestational diabetes mellitus (GDM) is increasingly common in pregnancy. This study's purpose was to identify the expression of XIST and manifest the potential mechanism of XIST in GDM.

Methods: Ninety-three patients with GDM and 93 normal pregnant women were included in this investigation. qRT-PCR was conducted to evaluate the expression of miR-497-5p and XIST and the relationship between XIST and fasting blood glucose (FBG) was explored by Pearson assay. The clinical diagnosis of XIST on GDM patients was validated by the receiver operator characteristic (ROC) curve. Cell counting kit-8 (CCK-8) was applied to elucidate cell viability. Luciferase reporter assay was performed to document the relationship among XIST, miR-497-5p, and FOXO1.

Results: The expression of XIST was increased in GDM patients and HTR-8/SVneo cell models caused by high glucose (HG). The expression of XIST was associated with the FBG levels and appeared to be a feasible indicator in discriminating GDM patients. The expression of miR-497-5p was prominently reduced in GDM patients and cell models. Inhibition of XIST might alleviate the adverse function of HG on cell viability via sponging miR-497-5p. FOXO1 was proved to be a downstream target gene of miR-497-5p.

Conclusions: Overexpression of XIST and downregulation of miR-497-5p were indicated in this publication. XIST might serve as a promising diagnostic marker for GDM patients. XIST/miR-497-5p/ FOXO1 axis played a critical role in the regulation of trophoblast cells.

Keywords: XIST/miR-497-5p/FOXO1; gestational diabetes mellitus (GDM); diagnosis; cell viability

Submitted Feb 23, 2021. Accepted for publication Apr 05, 2021.

doi: $10.21037 / \mathrm{cdt}-21-110$

View this article at: http://dx.doi.org/10.21037/cdt-21-110

\section{Introduction}

Gestational diabetes mellitus (GDM) is a health concern with anomalous glucose tolerance in pregnant women and a prevailing endocrine disorder (1). The insulin resistance and variation of fat metabolism and glycometabolism in the gestation period triggered by hormones and cytokines secreted by the placenta are a primary explanation of GDM (2). The possible risks of suffering cardiovascular diseases and type 2 diabetes for patients with a history of GDM are raised as previous investigations $(3,4)$. Meanwhile, GDM might lead to far-reaching problems for newborns and offspring. The children of GDM patients have a raised future risk of undergoing inflammation reaction, obesity, hypothalamus 
dysplasia, hyperactivity disorder, and neuropsychiatric morbidity (5-8). High glucose may induce the dysfunction of the placenta, which in turn affects fetal health and even leads to fetal death $(9,10)$. The normalization and stabilization of placenta and trophoblastic cells were fundamental in the duration of pregnancy for the health of unborn fetuses (11). As a result, opportunely and valid diagnosis and understanding of maternal GDM nosogenesis are beyond doubt to prevent the adverse effects of GDM on parturients and children.

Plenty of subjects has focused on the dysregulation of lncRNA in the GDM and trophoblastic cells. A microarray technology reveals that five underlying lncRNAs are closely relative to the GDM and fetal macrosomia and show a diagnostic ability for this sort of patient (12). An article published in 2018 is another evidence, which proposes that lncRNA MALAT1 highly expresses in the serum from GDM patients and differentiates GDM patients in early diagnosis (13). Moreover, the function of lncRNAs in trophoblast cells is an equally important aspect in the study of GDM. By way of illustration, Wang et al. tests the function of lncRNA PVT1 on the HTR8/SVneo cells and finds that PVT1 is dysregulated in the trophoblast cells and sustains normal biological function of trophoblast cells via regulating cell proliferation and movements (14). LncRNA X inactivatespecific transcript (XIST) is reported to contribute to diverse disorders, including diabetes mellitus. An achievement written by Zheng et al. mentions the dysregulation of XIST suppresses cell growth, inhibits cell migration, and represses cell invasion by regulating miR-155 and CDX1 gene (15). More importantly, the adhibition of the TaqMan Assay exemplifies that the expression of XIST is obviously raised in diabetic patients, emphasizing the importance of XIST in the clinical effect and progression of diabetes mellitus (16). Nonetheless, the expression pattern and function of XIST in GDM remains indeterminate.

Accordingly, our research aimed to identify the expression level of XIST in GDM patients and cell models of GDM. The diagnostic value of XIST for the case distinguishment was pinpointed in this report. Beyond these conclusions, the function of XIST on the viability of trophoblast cells and its target tool were determined in our findings.

We present the study in accordance with the STARD reporting checklist (available at http://dx.doi.org/10.21037/ cdt-21-110).

\section{Methods}

The study was conducted in accordance with the Declaration of Helsinki (as revised in 2013). All these people signed written informed consent before participating in this study and the Shanxi Provincial People's Hospital Ethics Committee provided support and approval to this design (approval no.2018243).

\section{Volunteers and experimental sample}

There were 93 patients with GDM and 93 healthy gestational women randomly included in this experiment and all these participants were from Shaanxi Provincial People's Hospital between May 2018 to June 2020. Based on the universal diagnostic standard, patients were determined as GDM after professional examinations (17). Some individuals who had a diabetic history before this pregnancy were not allowed to be included in our research. The fasting blood specimens were collected during the 24-28 weeks after pregnancy. A centrifugal machine was used to separate blood components and obtain a serum sample.

\section{Cell culture and transfection}

Human villous trophoblast cells HTR-8/SVneo were purchased from Procell company (Wuhan, China). The culture medium was RPMI-1640 (BioTek company, Beijing, China) including $1 \%$ antibiotic mixture and $5 \%$ FBS at $37^{\circ} \mathrm{C}$. The GDM cell models were built by culturing cells in HG medium which included $25 \mathrm{nM}$ glucose and control cells were incubated in a medium with $5 \mathrm{nM}$ glucose.

The si-XIST and its negative control (si-NC) together with miR-495-5p mimic (miR-mimic), miR-495-5p inhibitor (miR-inhibitor), and their negative control (miRNC) were all obtained from Sangon (Shanghai, China). The transfection experiments were in accordance with the specification of Lipofectamine 3000 (ThermoFisher, Waltham, MA, USA).

\section{Real-time quantitative PCR (qRT-PCR)}

TRIzol reagent was purchased to extracted total RNA from serum specimens and HTR-8/SVneo cells. The amount of total RNA was estimated using RNA HS Assay Kit (Vazyme, Nanjing, China) on Qubit 3.0 (ThermoFisher, Waltham, MA, USA) and purity of total RNA was figured out by NanoDrop 2000 (ThermoFisher, Waltham, MA, USA). Reverse transcription was performed on RNA using 
Table 1 Clinical features of healthy population and GDM patients

\begin{tabular}{lccc}
\hline Clinical features & Healthy population $(\mathrm{n}=93)$ & GDM patients $(\mathrm{n}=93)$ & $\mathrm{P}$ value \\
\hline Age (years) & $32.15 \pm 3.53$ & $32.34 \pm 3.80$ & 0.720 \\
BMI $\left(\mathrm{kg} / \mathrm{m}^{2}\right)$ & $21.29 \pm 2.87$ & $22.07 \pm 3.51$ & 0.097 \\
Pregnancy weeks (weeks) & $25.01 \pm 1.33$ & $24.90 \pm 1.40$ & 0.592 \\
FBG $(\mathrm{mM} / \mathrm{L})$ & $4.35 \pm 0.14$ & $6.20 \pm 0.13$ & $<0.001$ \\
\hline
\end{tabular}

GDM, gestational diabetes mellitus; BMI, body mass index; FBG, fasting blood glucose.

One Step miRNA cDNA Synthesis Kit (HaiGene, Harbin, China) or TaKaRa RT reagent Kit (Dalian, China).

The obtained cDNA was mixed with SYBR Premix Ex Taq kit (TaKaRa, Dalian, China) to determine the relative expression on the ABI 7500 system (ThermoFisher, Waltham, MA, USA). As internal references, the expression of GAPDH and U6 was measured. The primers of XIST were as follows: forward 5'-AGCTCCTCGGACAGCTGTAA-3' and reverse 5'-CTCCAGATAGCTGGCAACC-3'. The forward primer of miR-497-5p was 5 '-CCTTCAGCAGCACACTGTGG-3' and the revers primer was 5'-CAGTGCAGGGTCCGAGGTAT-3'. The $2^{-\Delta \Delta \mathrm{Ct}}$ method was applied to calculate the relative expression of XIST and miR-497-5p based on the Ct values of the realtime system. Each experiment was conducted at least 3 times.

\section{CCK-8 assay}

The cells during the growing period were resuspended in the trypsin and seeded into a 96-well plate. The concentration of cells in each well was adjusted to $1 \times 10^{3}$. After incubation at $0,1,2$, and $3 \mathrm{~d}, 10 \mu \mathrm{L}$ CCK-8 solution (Jiancheng, Nanjing, China) was mixed in the cell suspension and cultured for further 2 hours. After that, the OD data were detected under $450 \mathrm{~nm}$ with a microplate reader.

\section{Luciferase reporter assay}

A double-luciferase reporter assay kit from Transgen Biotech (Beijing, China) was obtained to detect luciferase activity. Namely, luciferase reaction substrate and substrate II were dissolved or diluted with the corresponding buffer. $1 \times$ cell lysis buffer was used to dissociate cells. Then, a total of $20 \mu \mathrm{L}$ cell lysate was mixed with $100 \mu \mathrm{L}$ luciferase reaction reagent. The luciferase activity was detected via a luminometer. About $100 \mu \mathrm{L}$ luciferase reaction reagent II was added into the mentioned tube and luciferase data were measured using the same method.

\section{Statistical analysis}

The data of our experiments were calculated in SPSS 12.0 and GraphPad 7.0. The comparisons of various groups were analyzed with a $t$-test, one-way ANOVA, and $\chi^{2}$ test. Pearson test was used to determine the correlation between the levels of XIST and fasting blood glucose (FBG) levels. The ROC curve was conducted to analyze the diagnostic value of XIST for GDM patients. The results were exhibited as mean $\pm \mathrm{SD}$. $\mathrm{P}$ values below 0.05 were considered significant.

\section{Results}

\section{The baseline data of the included population}

In order to understand the detailed information of all individuals who were recruited in our study, the clinical measurements and demographic features were collected and expressed in Table 1. As shown, ninety-three healthy populations with a mean age of $32.15 \pm 3.53$ years old and 93 GDM patients (mean age of $32.34 \pm 3.80$ ) years old were admitted in this experiment. No significant comparisons between controls and patients with GDM on age, body mass index (BMI), and pregnancy weeks were displayed in Table 1 $(\mathrm{P}>0.05)$. Conversely, the average FBG level was apparently higher in the GDM group than that in the healthy group $(\mathrm{P}<0.001)$.

\section{The expression of XIST is raised in GDM patients}

The relative expression of XIST of all recruiters was detected in our study to demonstrated the expression pattern of XIST. The findings were shown in Figure 1A, which illustrated that the relative expression of XIST was 
A

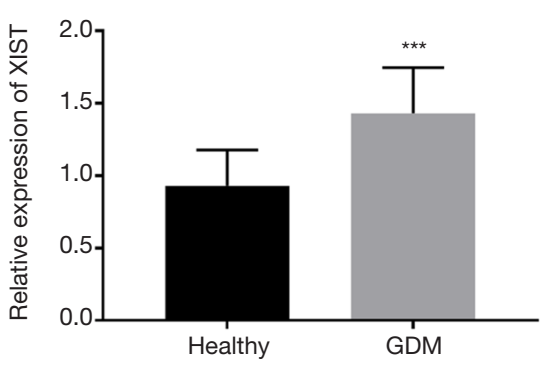

B

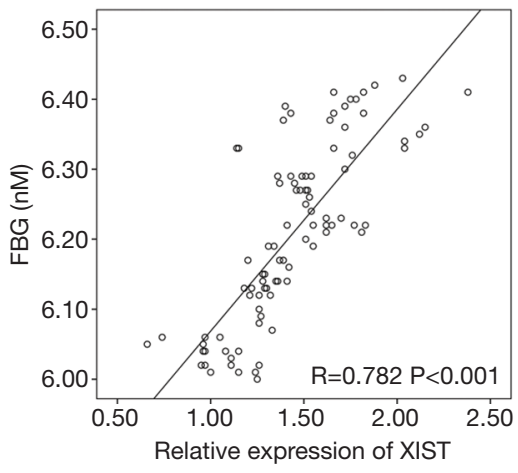

C



Figure 1 The levels and function of XIST in GDM. (A) The levels of XIST were increased in GDM patients. (B) The expression of XIST was positively related to the levels of FBG. (C) XIST showed a possibility as a diagnostic predictor with high sensitivity (0.806) and specificity $(0.860) .{ }^{* * *}, \mathrm{P}<0.001$, compared to healthy group. FBG, fasting blood glucose; GDM, gestational diabetes mellitus.


Figure 2 The levels and function of XIST in trophoblastic cell models induced by HG. (A) The expression of XIST was reduced by being transfecting with si-XIST in normal HTR-8/SVneo cells. (B) The transfection of si-XIST repressed the overexpression of XIST induced by HG. (C) Silence XIST reversed the increased OD values of viable cells induced by HG. ${ }^{* *}, \mathrm{P}<0.01$; ${ }^{* * *}, \mathrm{P}<0.001$, compared with control group. ", $\mathrm{P}<0.05$; \#\#, $\mathrm{P}<0.001$, compared with HG group. HG, high glucose.

conspicuously increased in GDM patients when compared to the healthy population $(\mathrm{P}<0.001)$. Furthermore, the correlation between relative levels of XIST and FBG was provided in Figure 1B. The levels of XIST were positively related to the concentration of FBG, indicating high levels of XIST participated in the development of GDM by rising FBG levels $(\mathrm{R}=0.782, \mathrm{P}<0.001)$.

\section{The diagnostic value of XIST for GDM patients}

Forasmuch as the aberrant abundance of XIST in patients with GDM, the forecasting value of XIST was elucidated. Figure $1 C$ disclosed that the area under the curve (AUC) of XIST was 0.897 together with, the sensitivity of 0.806 and the specificity of 0.860 at the cut-off value of 1.177 , suggesting XIST could differentiate GDM patients among healthy pregnant women.

\section{Silenced XIST inbibited the function of HG on cell viability}

To imitate the circumstances of GDM, the HG was used to culture trophoblastic cells and si-XIST was synthesized and transfected into HTR-8/SVneo cells to adjust the expression of XIST. In Figure $2 A$, the relative expression of XIST was distinctly decreased in the cells transfected with si-XIST as compared with control cells, proposing si-XIST could successfully silence XIST in HTR-8/SVneo cells $(\mathrm{P}<0.001)$.

As shown in Figure 2B, the relative expression of XIST was obviously increased in the cells treated with HG as compared with controls, whereas, this trend induced 

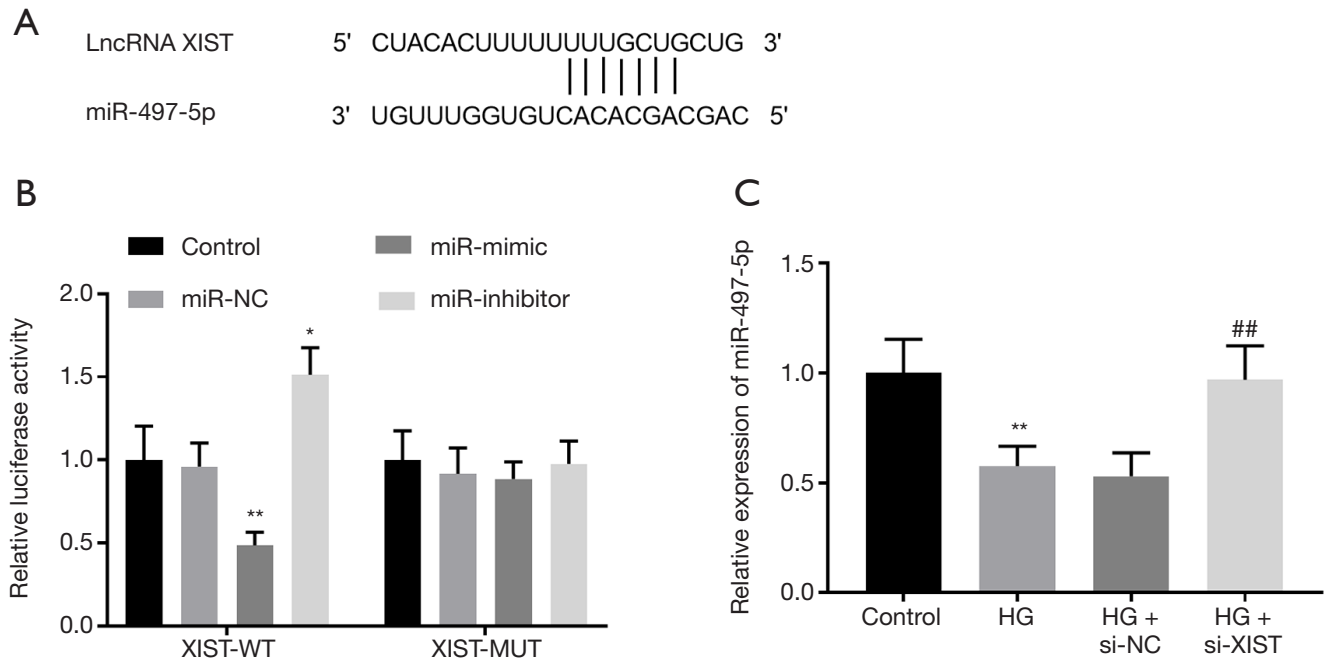

Figure 3 MiR-497-5p is a target of XIST. (A) The complementary bases of miR-497-5p and XIST. (B) The result of luciferase experiments. (C) The expression of miR-497-5p was negatively regulated by XIST. * $\mathrm{P}<0.05$; **, $\mathrm{P}<0.01$, compared with control group. ${ }^{\#}$, $\mathrm{P}<0.01$, compared with HG group. HG, high glucose.

by HG was conversed by the transfection of si-XIST $(\mathrm{P}<0.001)$. CCK-8 assay was carried out to examine the function of anomalous expression of XIST on cell viability. Experimental findings discovered that the viable count of cells in the HG group was remarkably decreased relative to the control group, and meanwhile, Figure $2 C$ figured out silenced XIST ameliorated the injury of HG on cell viability $(\mathrm{P}<0.05)$.

\section{XIST function as a ceRNA of miR-497-5p}

For examination of the inner molecular mechanism of XIST, StarBase was performed to predict the underlying regulatory relationship of XIST and miRNAs. In the predictive findings, XIST carried the complementary sequence of mature miR-497-5p, which was exhibited in Figure $3 \mathrm{~A}$. For further identification, a double luciferase reporter reaction was used to verify whether XIST directly bound miR-497-5p. As expected, the relative luciferase activity was reduced in the cells co-transfected with XIST-WT and miR-mimics compared to that in the cells transfected with XIST-WT only, and besides, the inhibition of miR-497-5p increased the luciferase activity (Figure $3 B$, $\mathrm{P}<0.05$ ). However, the luciferase activity had no difference among different treatments in the XIST-MUT group (Figure 3B, $\mathrm{P}>0.05$ ).

The relative expression of miR-497-5p was distinctly decreased in the HG group when compared with the control group, which was contrary to the expression of XIST in the HG group (Figure $3 C, \mathrm{P}<0.01$ ). Compared to the HG group, the downregulation of XIST increased the levels of miR-497-5p, reinforcing the results of luciferase reporter assay (Figure $3 C, \mathrm{P}<0.01$ ).

\section{The inhibition of miR-497-5p attenuated the influence of XIST on cell viability}

The expression of miR-497-5p was reduced in patients with GDM patients in contrast with healthy individuals, indicating that miR-497-5p might play a protective role in the progression of GDM (Figure $4 A, \mathrm{P}<0.001$ ). The miR497-5p inhibitors were transfected to regulate the relative expression of miR-497-5p. Compared to the control cells, the relative expression of miR-497-5p was decreased in the cells transfected with miR-497-5p inhibitors, indicating miR-497-5p inhibitors could induce the silence of miR497-5p (Figure 4B, $\mathrm{P}<0.01$ ).

Cells co-transfected with si-XIST and miR-497-5p inhibitors was detected the cell viability by CCK- 8 reaction. The results of miR-497-5p observed that the transfection with si-XIST and miR-497-5p inhibitors diminished the plenty of miR-497-5p caused by si-XIST (Figure $4 C$, $\mathrm{P}<0.01)$. Moreover, the low expression of miR-497-5p attenuated the high OD value on cell viability induced by si-XIST, announcing miR-497-5p might partake in the regulatory networks of XIST (Figure $4 D, \mathrm{P}<0.05$ ). 
A

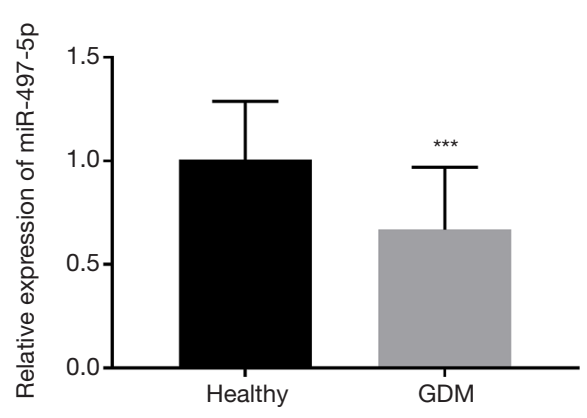

C

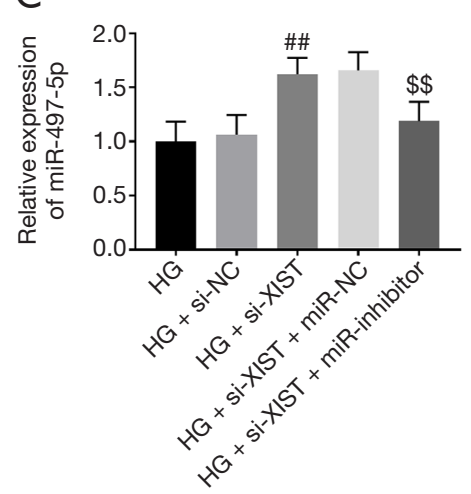

B



D



Figure 4 The expression and function of miR-497-5p in GDM. (A) The levels of miR-497-5p were decreased in GDM patients. (B) The expression of miR-497-5p was controlled by transfection of miR-497-5p inhibitors into normal HTR-8/SVneo cells. (C) The expression of miR-497-5p was accommodated by different translation experiments. (D) Knockdown of miR-497-5p attenuated the low viable cell models induced by si-XIST. **, $\mathrm{P}<0.01$; ***, $\mathrm{P}<0.001$, compared with control group. ${ }^{\text {\#\#, }} \mathrm{P}<0.01$, compared with HG group. ${ }^{\$}, \mathrm{P}<0.05 ;{ }^{\text {ss }}, \mathrm{P}<0.01$, compared with HG + si-XIST group. GDM, gestational diabetes mellitus; HG, high glucose.

\section{FOXO1 is a target gene of miR-497-5p}

Targetscan was used to predict the probable target genes of miR-497-5p and the result was shown in Figure 5A, which providing nine complementary bases between FOXO 3'UTR and mature miR-579-5p. A dual-luciferase reporter system was applied to determine that FOXO1 was a direct target of miR-497-5p by the evidence that in the FOXO1WT group, the miR-497-5p mimics reduced the luciferase activity and oppositely, overexpression of miR-497-5p enhanced the activity (Figure $5 B, \mathrm{P}<0.01$ ).

\section{Discussion}

From an investigation of 2019, about 20\% of pregnant women in the world are affected by GDM (18). GDM is the most hackneyed chronic disease during pregnancy, which may give rise to short-term and long-range complications for both mother and later generations (19). Most GDM women may get a definite diagnosis in the early trimester of pregnancy, however, the accuracy of diagnosis depends on the difference of environment, the individual standards, and the diverse strategies (20). More importantly, there is a lack of a unified strategy for diagnosing GDM women worldwide, which makes the researches on GDM more imminent.

In recent ten years, lncRNAs have been one of the hottest fields of a multitude of mRNA researches. A great deal of studies has found that the relative expression and regulatory function of miRNA are manipulated by lncRNAs (21). Besides, GDM-associated lncRNAs is increasing got the attention of scholars and more subjects were established to excavate the possible values on clinical therapies of GDM patients (22). A report published in 2020 investigated umbilical cord blood of GDM patients and healthy pregnant women provides $256 \operatorname{lncRNAs}$ are aberrantly expressed in the 

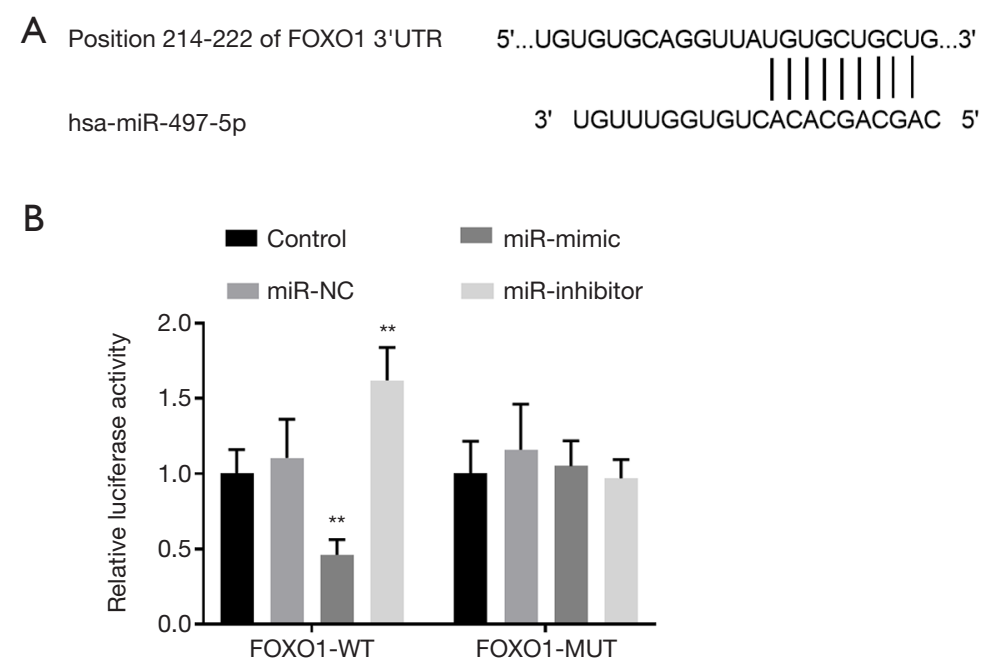

Figure 5 FOXO1 is a target gene of miR-497-5p. (A) The binding sites between FOXO1 and miR-497-5p. (B) Luciferase data confirmed FOXO1 is a target gene of miR-497-5p. **, $\mathrm{P}<0.01$, compared with control group.

exosomes from 3 patients with GDM compared to 3 healthy controls (23). An increased relative expression of MALAT1 was found in GDM patients and it can offer a promising predictor for GDM patients by the results of ROC (13). Conspicuously, accumulating lncRNAs was dysregulation in GDM and the latent function on identifying GDM patients was reported. In the current study, the expression of XIST was distinctly increased in maternal blood from GDM patients, and the abundance of XIST was accompanied by the increased FBG measurements. Furthermore, the ROC result unveiled that XIST might function as a feasible index in diagnosing GDM patients. A previous publication written by Sathishkumar et al. also supports our findings by the information that the relative expression of XIST was raised in patients with type 2 diabetes (24). Overall, elevated levels of XIST might participate in GDM and predict a high risk of suffering GDM.

HG-induced surrounding around trophoblast cells aggravated the injury of cells, influenced cell viability, and further aroused dysfunction of cells (25). Multitudinous researches state the function of lncRNAs on trophoblast cell functions. Zhu et al. illustrate this point clearly with the investigation that upregulation of lncRNA CRNDE expedites the cell viability by sponging miR-1277 (26). Likewise, another exemplification regarding lncRNA MEG3, which proposes cells from GDM patients showed diminished cell viability and overexpression of MEG3 further promotes viable cell number induced by GDM (27). In our study, we confirmed that HG raised the expression of XIST, and transfection with si-XIST reduced the upregulation of XIST induced by HG. Besides, the suppressed XIST repressed the lowered cell viability caused by HG. Previous studies validate the function of XIST on diversity cell. For instance, in inflammatory MAC-T cells, overexpression of XIST exists in the cells with inflammation and knockdown of XIST moderate the viability of inflammatory cells (28). The reduced expression of XIST restricted cell viability in Lung cancer cells via binding miR-144-3p (29). In terms of the existing results and previous finding, we hypothesized XIST might play as an adverse role in GDM by weakening cell viability.

In the present study, the target miRNA and possible regulatory mechanism of XIST were explored. Our findings proposed miR-497-5p is a promising target of XIST. The expression of miR-497-5p was decreased in the cells treated with HG and knockdown XIST elevated the levels of miR497-5p, which further inferred XIST played as a ceRNA of miR-497-5p. The inner interaction between XIST and miR497-5p was manifested in hepatocellular carcinoma (30) and colorectal cancer (31), and the XIST/miR-497-5p axis plays essential roles in malignant tumors. Of note, the expression of miR-497-5p was evidently decreased in GDM patients. The expression of miR-497-5p is reduced in cases with diabetic nephropathy and it participates in the accommodation of IncRNA ANRIL in diabetic nephropathy (32). A hot map proposes that miR-497-5p is insufficiently expressed in GDM patients, but no available mechanisms are mentioned (33). In this study, we deduced silence XIST reversed the weakened 
cell viability induced by HG and Co-transfection of si-XIST and miR-497-5p inhibited the tendency, indicating miR-497$5 \mathrm{p}$ mediated the influence of XIST on trophoblastic cell.

Substantial demonstration states FOXO1 regulates considerable biological progressions and may provide a momentous path for the prevention of diseases (34). In our research, we forecast that FOXO1 might be a logical target of miR-497-5p as a result of the complementary bases between both. Furthermore, the results of luciferase experiments indicated miR-497-5p inhibitors elevated the relative luciferase activity and its mimics reduced the activity in FOXO1-WT, attesting FOXO1 was a direct target of miR-497-5p in GDM. Previous studies discover FOXO1 is closely relevant to maternal diabetes and function as essential elements in an intrauterine program (35). In a statement conducted by $\mathrm{Xu}$ et al., the expression of FOXO1 is increased in the GDM group, which is consistent with our findings (36). One of limitation of our study was the small number of participants.

Taken together, the expression of XIST was increased and the expression of miR-497-5p was reduced in the GDM patients and trophoblastic cell models induced by HG. The overexpression of XIST was positively relative to the high FBG levels and XIST might act as a satisfactory predictive marker for GDM patients. Knockdown of XIST could reverse the decreased cell viability induced by HG via miR-497-5p/FOXO1 axis. These findings emphasized the regulatory influence of the XIST/miR-497-5p/ FOXO1 axis in GDM and proposed a novel perspective in understanding the pathogenesis of GDM.

\section{Acknowledgments}

Funding: This study was funded by the project grant "The correlation study of type 2 diabetes related mitochondrial tRNA mutation and gestational diabetes" (No. 2020JQ-938).

\section{Footnote}

Reporting Checklist: The authors have completed the STARD reporting checklist. Available at http://dx.doi.org/10.21037/ cdt-21-110

Data Sharing Statement: Available at http://dx.doi. org/10.21037/cdt-21-110

Peer Review File: Available at http://dx.doi.org/10.21037/ cdt-21-110
Conflicts of Interest: All authors have completed the ICMJE uniform disclosure form (available at http://dx.doi. org/10.21037/cdt-21-110). The authors have no conflicts of interest to declare.

Ethical Statement: The authors are accountable for all aspects of the work in ensuring that questions related to the accuracy or integrity of any part of the work are appropriately investigated and resolved. The study was conducted in accordance with the Declaration of Helsinki (as revised in 2013). The study was approved by the Shanxi Provincial People's Hospital Ethics Committee (approval No. 2018243) and informed consent was collected from each participant.

Open Access Statement: This is an Open Access article distributed in accordance with the Creative Commons Attribution-NonCommercial-NoDerivs 4.0 International License (CC BY-NC-ND 4.0), which permits the noncommercial replication and distribution of the article with the strict proviso that no changes or edits are made and the original work is properly cited (including links to both the formal publication through the relevant DOI and the license). See: https://creativecommons.org/licenses/by-nc-nd/4.0/.

\section{References}

1. ACOG Practice Bulletin No. 190: Gestational Diabetes Mellitus. Obstet Gynecol 2018;131:e49-64.

2. Świrska J, Zwolak A, Dudzińska M, et al. Gestational diabetes mellitus - literature review on selected cytokines and hormones of confirmed or possible role in its pathogenesis. Ginekol Pol 2018;89:522-7.

3. Kramer CK, Campbell S, Retnakaran R. Gestational diabetes and the risk of cardiovascular disease in women: a systematic review and meta-analysis. Diabetologia 2019;62:905-14.

4. Moon JH, Kwak SH, Jang HC. Prevention of type 2 diabetes mellitus in women with previous gestational diabetes mellitus. Korean J Intern Med 2017;32:26-41.

5. Antikainen L, Jääskeläinen J, Nordman H, et al. Prepubertal Children Exposed to Maternal Gestational Diabetes Have Latent Low-Grade Inflammation. Horm Res Paediatr 2018;90:109-15.

6. Page KA, Luo S, Wang X, et al. Children Exposed to Maternal Obesity or Gestational Diabetes Mellitus During Early Fetal Development Have Hypothalamic Alterations That Predict Future Weight Gain. Diabetes Care 
2019;42:1473-80.

7. Xiang AH, Wang X, Martinez MP, et al. Maternal Gestational Diabetes Mellitus, Type 1 Diabetes, and Type 2 Diabetes During Pregnancy and Risk of ADHD in Offspring. Diabetes Care 2018;41:2502-8.

8. Nahum Sacks K, Friger M, Shoham-Vardi I, et al. Prenatal exposure to gestational diabetes mellitus as an independent risk factor for long-term neuropsychiatric morbidity of the offspring. Am J Obstet Gynecol 2016;215:380.e1-7.

9. Hulme CH, Nicolaou A, Murphy SA, et al. The effect of high glucose on lipid metabolism in the human placenta. Sci Rep 2019;9:14114.

10. Carrasco-Wong I, Moller A, Giachini FR, et al. Placental structure in gestational diabetes mellitus. Biochim Biophys Acta Mol Basis Dis 2020;1866:165535.

11. Stirm L, Kovárová M, Perschbacher S, et al. BMIIndependent Effects of Gestational Diabetes on Human Placenta. J Clin Endocrinol Metab 2018;103:3299-309.

12. Lu J, Wu J, Zhao Z, et al. Circulating LncRNA Serve as Fingerprint for Gestational Diabetes Mellitus Associated with Risk of Macrosomia. Cell Physiol Biochem 2018;48:1012-8.

13. Zhang $\mathrm{Y}, \mathrm{Wu} \mathrm{H}$, Wang $\mathrm{F}$, et al. Long non-coding RNA MALAT1 expression in patients with gestational diabetes mellitus. Int J Gynaecol Obstet 2018;140:164-9.

14. Wang Q, Lu X, Li C, et al. Down-regulated long noncoding RNA PVT1 contributes to gestational diabetes mellitus and preeclampsia via regulation of human trophoblast cells. Biomed Pharmacother 2019;120:109501.

15. Zheng R, Lin S, Guan L, et al. Long non-coding RNA XIST inhibited breast cancer cell growth, migration, and invasion via miR-155/CDX1 axis. Biochem Biophys Res Commun 2018;498:1002-8.

16. Sohrabifar N, Ghaderian SMH, Alipour Parsa S, et al. Variation in the expression level of MALAT1, MIAT and XIST lncRNAs in coronary artery disease patients with and without type 2 diabetes mellitus. Arch Physiol Biochem 2020. [Epub ahead of print]. doi: 10.1080/13813455.2020.1768410.

17. American Diabetes Association. 2. Classification and Diagnosis of Diabetes: Standards of Medical Care in Diabetes-2018. Diabetes Care 2018;41:S13-27.

18. Yang S, Lin R, Si L, et al. Cod-Liver Oil Improves Metabolic Indices and hs-CRP Levels in Gestational Diabetes Mellitus Patients: A Double-Blind Randomized Controlled Trial. J Diabetes Res 2019;2019:7074042.

19. Franzago M, Fraticelli F, Stuppia L, et al. Nutrigenetics, epigenetics and gestational diabetes: consequences in mother and child. Epigenetics 2019;14:215-35.

20. Immanuel J, Simmons D. Screening and Treatment for Early-Onset Gestational Diabetes Mellitus: a Systematic Review and Meta-analysis. Curr Diab Rep 2017;17:115.

21. Zhao Z, Sun W, Guo Z, et al. Mechanisms of lncRNA/ microRNA interactions in angiogenesis. Life Sci 2020;254:116900.

22. Leng L, Zhang C, Ren L, et al. Construction of a long non-coding RNA-mediated competitive endogenous RNA network reveals global patterns and regulatory markers in gestational diabetes. Int J Mol Med 2019;43:927-35.

23. Cao M, Zhang L, Lin Y, et al. Differential mRNA and Long Noncoding RNA Expression Profiles in Umbilical Cord Blood Exosomes from Gestational Diabetes Mellitus Patients. DNA Cell Biol 2020;39:2005-16.

24. Sathishkumar C, Prabu P, Mohan V, et al. Linking a role of lncRNAs (long non-coding RNAs) with insulin resistance, accelerated senescence, and inflammation in patients with type 2 diabetes. Hum Genomics 2018;12:41.

25. Zhang C, Wang L, Chen J, et al. Differential Expression of miR-136 in Gestational Diabetes Mellitus Mediates the High-Glucose-Induced Trophoblast Cell Injury through Targeting E2F1. Int J Genomics 2020;2020:3645371.

26. Zhu H, Kong L. LncRNA CRNDE regulates trophoblast cell proliferation, invasion, and migration via modulating miR-1277. Am J Transl Res 2019;11:5905-18.

27. Ye HH, Yang SH, Zhang Y. MEG3 damages fetal endothelial function induced by gestational diabetes mellitus via AKT pathway. Eur Rev Med Pharmacol Sci 2018;22:8553-60.

28. Ma M, Pei Y, Wang X, et al. LncRNA XIST mediates bovine mammary epithelial cell inflammatory response via NF-кB/NLRP3 inflammasome pathway. Cell Prolif 2019;52:e12525.

29. Tian LJ, Wu YP, Wang D, et al. Upregulation of Long Noncoding RNA (lncRNA) X-Inactive Specific Transcript (XIST) is Associated with Cisplatin Resistance in NonSmall Cell Lung Cancer (NSCLC) by Downregulating MicroRNA-144-3p. Med Sci Monit 2019;25:8095-104.

30. Zhang Y, Zhu Z, Huang S, et al. lncRNA XIST regulates proliferation and migration of hepatocellular carcinoma cells by acting as miR-497-5p molecular sponge and targeting PDCD4. Cancer Cell Int 2019;19:198.

31. Wang N, He JX, Jia GZ, et al. The lncRNA XIST promotes colorectal cancer cell growth through regulating the miR-497-5p/FOXK1 axis. Cancer Cell Int 2020;20:553.

32. Wang J, Zhao SM. LncRNA-antisense non-coding 
RNA in the INK4 locus promotes pyroptosis via miR497/thioredoxin-interacting protein axis in diabetic nephropathy. Life Sci 2021;264:118728.

33. Zhu Y, Tian F, Li H, et al. Profiling maternal plasma microRNA expression in early pregnancy to predict gestational diabetes mellitus. Int J Gynaecol Obstet 2015;130:49-53.

34. Xing YQ, Li A, Yang Y, et al. The regulation of FOXO1 and its role in disease progression. Life Sci

Cite this article as: Li Y, Yuan X, Shi Z, Wang H, Ren D, Zhang Y, Fan Y, Liu Y, Cui Z. LncRNA XIST serves as a diagnostic biomarker in gestational diabetes mellitus and its regulatory effect on trophoblast cell via miR-497-5p/FOXO1 axis. Cardiovasc Diagn Ther 2021;11(3):716-725. doi: 10.21037/ cdt-21-110
2018;193:124-31.

35. Chen C, Luo Y, Su Y, et al. The vitamin D receptor (VDR) protects pancreatic beta cells against Forkhead box class O1 (FOXO1)-induced mitochondrial dysfunction and cell apoptosis. Biomed Pharmacother 2019;117:109170.

36. $\mathrm{Xu} \mathrm{Y,} \mathrm{Jin} \mathrm{B,} \mathrm{Sun} \mathrm{L,} \mathrm{et} \mathrm{al.} \mathrm{The} \mathrm{expression} \mathrm{of} \mathrm{FoxO1} \mathrm{in}$ placenta and omental adipose tissue of gestational diabetes mellitus. Exp Clin Endocrinol Diabetes 2014;122:287-94. 